criteria and a review stage. Each of these has further substages and it includes a focus on how the content of the process and the framework interacts with the consultation and involvement of patients, public and the wider staff.

Discussion To assess its impact, the four sites using the framework will be compared with the remaining 27 HSCP sites. The aim of the comparison is to establish: the extent to which the remaining sites use elements of the framework; the principles and processes used for decision-making, and whether decisions have resulted in evidence-based resource shifts.

\section{P20 ARE POLITICAL FEATURES ASSOCIATED WITH POPULATION HEALTH OUTCOMES? A SYSTEMATIC REVIEW OF INTERNATIONALLY COMPARATIVE STUDIES}

${ }^{1} \mathrm{M}$ Barnish*, ${ }^{2} \mathrm{M}$ Tørnes, ${ }^{3} \mathrm{~B}$ Horne. ${ }^{1}$ Institute for Health Research, University of Exeter, Exeter, UK; ${ }^{2}$ Ageing Clinical and Experimental Research (ACER) Team, University of Aberdeen, Aberdeen, UK; ${ }^{3} N / A$, Independent Scholar, London, UK

\subsection{6/jech-2018-SSMabstracts. 146}

Background There are strong structural and theoretical reasons to expect politics to be an important determinant of population health outcomes. However, the most recently available systematic review of the evidence linking key political features (welfare state generosity, political tradition along the left-right axis, democracy, and globalisation) and population health outcomes contains searches only up to April 2010. Considering only internationally comparative studies, it found preliminary evidence that pro-social political features predicted better population health, but more up to date evidence synthesis is required. Therefore, the aim of this study was to present an updated systematic review on the political determinants of population health.

Methods Ten academic bibliographical databases, including MEDLINE, EMBASE, and Sociological Abstracts, were searched using search terms based on ((democracy OR autocracy OR welfare regime OR welfare state OR welfare capitalism OR politics OR political tradition OR internationality OR globalization) AND (health OR health services OR population health OR public health OR health economics OR health expenditure)). Supplementary searches were also conducted on Google Scholar and in relevant bibliographies. The final search was conducted in November 2017. We considered full-text scholarly articles or book chapters assessing the relationship between at least one of our eligible political features (welfare state generosity, political tradition along the left-right axis, democracy, and globalisation) and any population health outcome in human populations, except healthcare spending. Standardised data extraction, risk of bias assessment and narrative synthesis were conducted. Proportionate second review was conducted.

Results 73 articles were identified from the previous 2010 review. Updated database searches yielded 43356 records (35 207 unique) and supplementary searches yielded 55. Fulltext screening was conducted on 255 publications, and 176 publications (176 studies) were included, of which 106 were newly identified by our 7-year update. 79/102 studies found that increased welfare state generosity predicted greater population health. 15/17 studies found this effect for left-of-centre political tradition, as did $34 / 44$ for democracy. Half of identified studies suggested that globalisation may be detrimental for health. 85 studies were at low risk of bias, 89 moderate, none high, and two could not be assessed.

Conclusion The evidence shows politics to be an important determinant of population health outcomes. The public health community should engage more with the political determinants of health in research and advocacy. Further research on globalisation is required. While we provide a rigorous and timely update, resources prevented us from conducting a new systematic review from inception.

\section{P21 THE HEALTH OF ADULTS WHO HAD BEEN IN CARE UP TO 40 YEARS EARLIER: ARE THERE DIFFERENCES BY TYPE OF CARE? FINDINGS FROM THE ONS LONGITUDINAL STUDY}

ET Murray*, R Lacey, A Sacker. Department of Epidemiology and Public Health, University College London, London, UK

\subsection{6/jech-2018-SSMabstracts. 147}

Background The adverse life-long consequences of being looked after as a child are well recognised. However, systematic evidence on outcomes for looked-after children beyond the early adult years is currently very limited.

Methods Data were used from »120000 dependent children (aged $<18$ and never-married) in the ONS Longitudinal Study (LS) in each of the census years 1971, 1981, 1991 and 2001 (total $n=495,165)$. Separately for each census, logistic regression was used to compare odds of long-term limiting illness and self-rated health 10,20 and 30 years later for LS members with different care experiences in childhood (parental household, relative household, non-relative household and residential care).

Results In this nationally-representative sample of children in England and Wales, approximately $1.4 \%$ of dependent children were looked after in non-parental households, 3664 children in relative households, 2351 in unrelated households and 1007 in residential care. From 1971 to 2001 , there was a general trend of an increasing percentage of dependent children residing in relative households $(0.4 \%$ to $1.2 \%)$ and decreasing percentage in residential care $(0.3 \%$ to $0.1 \%)(\mathrm{p}$-values $<0.001)$. At 10,20 and 30 year follow-up, LS members who had resided outside the parental home in childhood, had higher odds of a longterm limiting illness and rating their health as 'not good' vs 'good'; with the highest odds for residential care. For example, for LS members who were dependent children in 1991, odds of a long-term limiting illness 10 years later were 7.8 (95\% confidence interval: 5.3, 11.4) higher for those who had resided in residential care, 1.9 (1.4, 2.7) higher in relative households and $2.4(1.9,3.1)$ higher in non-relative households, compared to residence in a parental household. For non-relative and residential care, odds ratios increased with each successive census year. The longer the follow-up period the weaker the strength of association, but with all associations remaining significant. Weaker associations were seen for self-rated health compared to long-term limiting illness.

Additional analysis to be presented will make step-wise adjustments for childhood demographics and social circumstances. 
Conclusion Decades after children and youths are placed in out-of-home care; they are still likely to report worse health than children who grew up in a parental household. Future work from this project will investigate how much of these associations are due to socio-demographic selection into different care experiences, compared to effects of being in care.

\section{P22 ASSOCIATIONS BETWEEN SOCIAL CAPITAL AND MENTAL HEALTH IN TWO RUSSIAN CITIES}

${ }^{1} \mathrm{~N}$ Bobrova*, ${ }^{1} \mathrm{~S}$ Cook, ${ }^{2} \mathrm{AV}$ Kudryavtsev, ${ }^{3,4} \mathrm{~S}$ Malyutina, ${ }^{3} \mathrm{M}$ Voevoda, ${ }^{1,5} \mathrm{DA}$ Leon. ${ }^{1}$ Department of Non-communicable Disease Epidemiology, London School of Hygiene and Tropical Medicine, London, UK; ${ }^{2}$ Central Scientific Research Laboratory, Northern State Medical University, Arkhangelsk, Russia; ${ }^{3}$ Research Institute of Internal and Preventive Medicine - Branch of ICandG SB RAS, Novosibirsk, Russia; ${ }^{4}$ Novosibirsk State Medical University, Ministry of Health of Russia, Novosibirsk, Russia; ${ }^{5}$ Department of Community Medicine, Arctic University of Norway, UiT, Tromso, Norway

\subsection{6/jech-2018-SSMabstracts. 148}

Background Social capital has been extensively studied as one of the determinants of population health including mental health over the past 20 years. There is existing evidence linking depressive disorders with CVD mortality and all-cause mortality in many countries including Russia. However, research exploring determinants of mental health in Russia including social capital is limited.

Methods We assessed relationships between social capital and mental health in a large cross-sectional study, the International Project on Cardiovascular Disease in Russia (IPCDR), based in two Russian cities: Arkhangelsk and Novosibirsk. The study used a random sample of 5051 people aged 35-69. The collected data included measures of socio-economic status and demographic variables: age, sex, education, perceived financial situation, and marital status.

Mental health measures Having any depressive or anxiety disorder were defined as $\geq 10$ score calculated from the Patient Health Questionnaire (PHQ-9) and Generalized Anxiety Disorder 7 (GAD-7) scales.

Social capital measures We used three questions to measure social capital with 11-point scale answer options: 1) Social mistrust, 'Generally speaking, would you say that most people can be trusted, or that you can't be too careful in dealing with people?'; 2) Perceived lack of fairness: 'Do you think that most people would try to take advantage of you if they got the chance, or would they try to be fair?'; and 3) Perceived unhelpfulness: 'Would you say that most of the time people try to be helpful or that they are mostly looking out for themselves'. The scales were dichotomised 0/6-low social capital and 7/10-high social capital and each was used as a separate dependent variable in analysis.

Statistical analysis We employed two logistic regression models to assess associations between social capital and mental health, 1) adjusting for age, sex and city, 2) adjusting for age, sex, city, marital status, education level and self-reported financial situation.

Results All three measures of social capital were associated with depression and anxiety after adjusting for socio-economic and demographic variables: 'Social mistrust': OR 1.49 (95\% CI, 1.14 to 1.94), 'Perceived lack of fairness': OR 1.75 (95\% CI, 1.39 to 2.20), 'Perceived unhelpfulness' OR 1.72 (95\% CI, 1.36 to 2.17) for depressive disorder; 'Social mistrust': OR 1.40 (95\% CI, 0.99 to 1.99), 'Perceived lack of fairness':
OR 2.37 (95\% CI, 1.71 to 3.29), and 'Perceived unhelpfulness': OR 2.19 (95\% CI, 1.57 to 3.04) for anxiety disorder. Conclusion Our findings from Russia support previous research showing the relationships between social capital and mental health. Further investigations on direction of the effect and pathways explaining these associations are needed.

\section{P23 'POSITIVE AND NEGATIVE SOCIAL SUPPORT AND HPA- AXIS HYPERACTIVITY: EVIDENCE FROM GLUCOCORTICOIDS IN HUMAN HAIR'}

${ }^{1} \mathrm{E}$ lob*, ${ }^{2} \mathrm{C}$ Kirschbaum, ${ }^{3} \mathrm{~A}$ Steptoe. ${ }^{1}$ Department of Epidemiology and Public Health, University College London, London, UK; ${ }^{2}$ Department of Psychology, Technische Universität Dresden, Dresden, Germany; ${ }^{3}$ Department of Behavioural Science and Health, University College London, London, UK

\subsection{6/jech-2018-SSMabstracts. 149}

Background While positive social support is associated with lower prevalence of disease and better treatment outcomes, negative social relationships can instead have unfavourable consequences for several physical and mental health conditions. However, the specific mechanisms by which this nexus might operate remain poorly understood. Hypothalamic-pituitary-adrenal (HPA) axis hyperactivity owing to psychosocial stress has been proposed as a potential pathway underlying the link between social support and health. Hair glucocorticoids such as cortisol and cortisone are emerging as promising biomarkers of long-term retrospective HPA activation. Therefore, the aim of this investigation was to examine the effects of positive and negative experiences of social support within key relationships (i.e. spouse/partner, children, other family members, and friends) on cortisol and cortisone.

Methods These associations were tested in a sample of 2520 older adults (mean age 68.1) from the English Longitudinal Study of Ageing (ELSA). Hair samples were collected in wave $6(2012 / 13)$. To understand the impact of cumulative exposure to poor social support, the analysis used self-reported data from waves $4(2008 / 09)$ and 6. Both aggregated and sourcespecific effects of social support were tested. Covariates included demographic characteristics, socioeconomic position, health behaviours, and hair characteristics. The analytical strategy was based on multiple linear regression, and missing data were estimated by multiple imputation.

Results In cross sectional analyses, participants who reported lower positive or greater negative social support across all sources had higher levels of cortisol, as well as an elevated cortisol-to-cortisone ratio. Amongst the different components of social support, children exhibited the largest effects on both hormones. In longitudinal analyses, cortisol and the ratio were positively associated with the aggregated scores for cumulative exposure to poor social support, evaluated in retrospect over four years.

Discussion Experiences of low positive and high negative social support, particularly from children, were both related to higher hair glucocorticoid levels. Hence, poorer quality of social relationships in later life may lead to higher psychosocial stress. This in turn could result in HPA-axis hyperactivity and increase the individual's susceptibility to poor health. Health and social care interventions should therefore highlight the importance of improving the quality of social relationships, rather than merely enhancing social interactions, in order to reduce stress and ameliorate health. 